\title{
Diagnostic hysteroscopy and saline infusion sonography in the diagnosis of intrauterine abnormalities: an assessment of patient preference
}

\author{
Heleen van Dongen • Anne Timmermans • \\ Cathrien E. Jacobi • Trudy Elskamp • \\ Cor D. de Kroon • Frank Willem Jansen
}

Received: 1 June 2010 / Accepted: 14 December 2010 /Published online: 30 December 2010

(C) The Author(s) 2010. This article is published with open access at Springerlink.com

\begin{abstract}
This study was conducted to assess whether women would prefer to undergo saline infusion sonography (SIS) or office hysteroscopy for the investigation of the uterine cavity. In a randomised controlled trial, 100 patients underwent SIS or office hysteroscopy for assessing patients' pain scores. After the investigation, 92 of them were asked to fill out an anonymous questionnaire addressing their preference regarding the method of evaluation and treatment of the uterine cavity. A control group, consisting of 50 women who never underwent SIS or office hysteroscopy, was also asked to complete an identical questionnaire. The questionnaire was completed by 113 women (83.7\%). Twenty-four (21.2\%) women would opt for SIS, whereas $52(46.0 \%)$ would opt for office hysteroscopy, and 37 $(32.7 \%)$ had no preference. If therapy would be necessary, $48.7 \%$ of the women would opt for an outpatient treatment, whereas $33.0 \%$ of the women would prefer treatment under general anaesthesia. Despite the fact that SIS is less painful, the majority of the women prefer office hysteroscopy.
\end{abstract}

H. van Dongen $(\bowtie) \cdot$ T. Elskamp $\cdot$ C. D. de Kroon $\cdot$ F. W. Jansen Department of Gynaecology, Leiden University Medical Center, Albinusdreef 2,

2300 RC Leiden, The Netherlands

e-mail: H.van_Dongen@lumc.nl

A. Timmermans

Department of Gynaecology, University Medical Center Utrecht, Utrecht, The Netherlands

C. E. Jacobi

Department of Medical Decision Making,

Leiden University Medical Center,

Leiden, The Netherlands
Additionally, therapy in an outpatient setting is preferred to a day case setting.

Keywords Preference - Intrauterine abnormalities . Hysteroscopy · Saline infusion sonography - Diagnosis . Outpatient

\section{Background}

Hysteroscopy is widely accepted to be the accurate standard for investigation of the uterine cavity [1, 2]. A metaanalysis showed that the diagnostic accuracy of saline infusion sonography (SIS) equals the accuracy of diagnostic hysteroscopy [3]. With the introduction of SIS, several authors showed that SIS provided less discomfort for patients compared to office hysteroscopy $[4,5]$. However, since the development of smaller diameter hysteroscopic systems and the introduction of a 'vaginoscopic' approach to hysteroscopy (without speculum and tenaculum), patient compliance has improved considerably [6]. A great advantage of hysteroscopy over SIS is the possibility to perform a directed biopsy or a small surgical intervention in the same session. Moreover, gynaecological treatments in an outpatient setting have shown to be highly acceptable and popular with patients $[7,8]$.

Nevertheless, we recently showed in a randomised comparison of outpatient vaginoscopic hysteroscopy and SIS that the latter still is significantly less discomforting [9]. To follow up on this, we undertook a survey to determine which form of investigation (SIS or office hysteroscopy) women prefer and for what reasons. 


\section{Methods}

This study was conducted at the Department of Gynaecology of Leiden University Medical Center (Leiden, The Netherlands) from January 2006 to July 2007. One hundred and forty-two women were approached to complete a questionnaire. Ninety two of these women were included in a randomised controlled trial comparing the pain scores of SIS and office hysteroscopy performed according to the vaginoscopic approach [9]. These women were randomly allocated to SIS or office hysteroscopy (solely for diagnostic purposes) and underwent one of these procedures. They were therefore not influenced by their doctor's choice or their own preferences. They received the questionnaire following the allocated investigation.

Another 50 women attending the general gynaecology outpatient clinic, for reasons other than the investigation of the uterine cavity, were randomly asked by their doctor to complete the questionnaire as well. All the women attending the outpatient clinic were eligible to participate in this control group irrespective of age, parity, general health or menopausal status. If they had previously undergone SIS or office hysteroscopy, they were excluded from analysis. Ethical approval for this study was obtained from the Leiden University Medical Center Ethics Committee.

\section{Questionnaire}

A questionnaire was designed and consisted of a general part and a problem-specific part. The general part addressed demographic data, including employment and number of children, and a brief medical history, including questions relating to the patient's past experience with outpatient procedures (e.g. colposcopy or mini-curettage). It also addressed the patients' preferred role in the decisionmaking process, including to what extent they would like to be informed about advantages and disadvantages.

The problem-specific part focused on the preference of SIS or hysteroscopy. The primary outcome measures of this questionnaire were preference for SIS or hysteroscopy in a diagnostic setting and preference for therapy in an outpatient or day case setting. Secondary outcome measures were factors influencing preference.

For this purpose, a full description with advantages and disadvantages of both investigations was provided (Appendix A). In an attempt to minimise bias, the descriptions were based on patient information of the Dutch Society of Obstetrics and Gynaecology. The order of the descriptions was randomly allocated. With reference to these descriptions, women were asked to state which of the two investigations they would choose if they needed an investigation of the uterine cavity (again) and what was most important in their decision and preferences, scored on a Likert scale. Since controls had no experience with any of the investigations, these questions were rewritten for better understanding. Following this, women were specifically asked to what extent they would accept pain in exchange for certain advantages of the investigations and what the required benefit should be in terms of successful therapy. In addition, they were asked what level of pain they would expect to experience during both investigations, scored on a visual analogue scale (VAS) of $10 \mathrm{~cm}$. Finally, they were asked in what setting (i.e. outpatient without anaesthesia or day case with general or regional anaesthesia) they would prefer treatment if during the investigation an abnormality would be found. To maximise the response rate, a reminder was sent by mail to the non-responders after 6 weeks.

The received information was entered in statistical software (SPSS, version 14; SPSS Inc., Chicago, IL). Patient characteristics of the study groups were analysed with one-way ANOVA in case of normally distributed, continuous variables and Pearson's chi-square test in case of dichotomous data. Confidence intervals for difference in means were calculated. Pearson's chi-square was also used to analyse differences of preference among the study groups. A paired-samples $t$ test was used to compare expected VAS scores for both investigations. All tests were two-sided, and $p$ values $<0.05$ were considered statistically significant.

\section{Findings}

Of the 142 women approached, 113 (79.6\%) women completed the questionnaire: 79 in the trial and 34 in the control group. Of the trial patients, $38(33.6 \%)$ underwent SIS and 41 (36.3\%) underwent office hysteroscopy (Fig. 1).

Table 1 presents data on the personal characteristics of the participating women, stratified by study group. Twentyfour $(21.2 \%)$ women would opt for SIS, whereas 52 (46.0\%) would opt for office hysteroscopy, and 37 (32.7\%) had no preference. Because preferences were strongly related to previous investigation, we present them by group as well (Fig. 2). The percentage of women preferring SIS or hysteroscopy differs significantly when women are stratified by the allocated investigation (hysteroscopy or SIS; Pearson's Chi-square, $p=0.003$ ). There were no differences found by social characteristics when stratified according to preference. Women preferring SIS expected to experience significantly less pain during SIS than during hysteroscopy (VAS scores 3.0 and 4.9 , respectively; $p=0.006$ ), whereas women preferring hysteroscopy expected to experience similar pain levels during both investigations (VAS scores 3.1 and 3.5 for SIS and hysteroscopy, respectively; $p=0.120$ ). There were no significant differences of pain scores measured during the investigation the patients were allocated to (data from van Dongen et al. [9]) with regard to their postexamination 
Fig. 1 Flowchart depicting received procedures and participation in preference study of women participating in randomised trial comparing pain scores

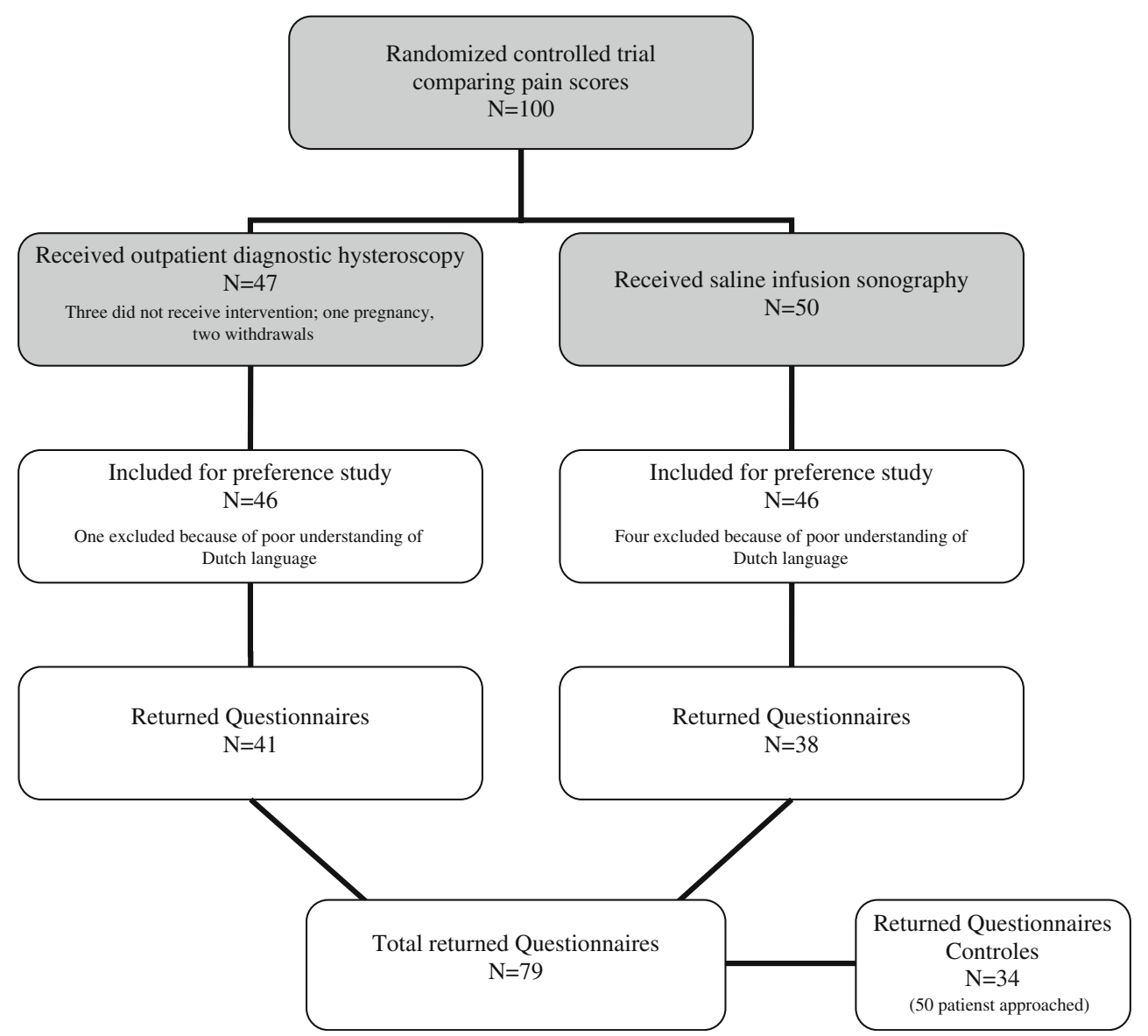

preferences (Table 2). The pain levels measured during both examinations differed significantly in favour of SIS [9].

Different aspects of preferences with regard to the investigation of the uterine cavity and possible necessitating therapy were scored on a Likert scale $(0=$ unimportant, $5=$ very important) and are detailed in Table 3. Except for having or not having anaesthesia during therapy, every aspect was considered important. When stratifying the
Likert scores by preference (SIS or office hysteroscopy), the following differences were found. Women with a preference for office hysteroscopy found it of utmost importance that diagnosis and therapy are offered in one visit ( $p=0.004)$, whereas women preferring SIS felt time to consider treatment options more important than women preferring office hysteroscopy $(p=0.014)$. With regard to what extent women would accept pain in exchange for

Table 1 Patient characteristics stratified by study group

\begin{tabular}{|c|c|c|c|c|}
\hline Outcome & SIS $(n=38)$ & Office hysteroscopy $(n=41)$ & Controls $(n=34)$ & $p$ value \\
\hline Age in years $(95 \%-\mathrm{CI})$ & $45.0(41.8-48.2)$ & $44.7(42.3-47.1)$ & $42.2(37.5-47.0)$ & $0.484^{\mathrm{a}}$ \\
\hline Employment & $28(73.7 \%)$ & $32(80.0 \%)$ & $28(82.4 \%)$ & $0.901^{\mathrm{b}}$ \\
\hline Married/living together & $30(78.9 \%)$ & $34(82.9 \%)$ & $28(82.4 \%)$ & $0.795^{\mathrm{b}}$ \\
\hline Children & $30(78.9 \%)$ & $29(70.7 \%)$ & $20(58.8 \%)$ & $0.176^{\mathrm{b}}$ \\
\hline Accessibility of hospital & $35(92.1 \%)$ & $40(97.6 \%)$ & $32(94.1 \%)$ & $0.215^{\mathrm{b}}$ \\
\hline Premenopausal & $31(81.6 \%)$ & $34(82.9 \%)$ & $25(73.5 \%)$ & $0.564^{\mathrm{b}}$ \\
\hline Postmenopausal & $7(18.4 \%)$ & $7(17.1 \%)$ & $9(26.5 \%)$ & $0.564^{\mathrm{b}}$ \\
\hline Previous surgery & $31(81.6 \%)$ & $32(78.0 \%)$ & $26(76.5 \%)$ & $0.808^{\mathrm{b}}$ \\
\hline Previous intervention in outpatient setting & $23(60.5 \%)$ & $29(70.7 \%)$ & $21(61.8 \%)$ & $0.477^{\mathrm{b}}$ \\
\hline
\end{tabular}

SIS saline infusion sonography

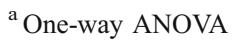

${ }^{\mathrm{b}}$ Pearson's Chi-square 


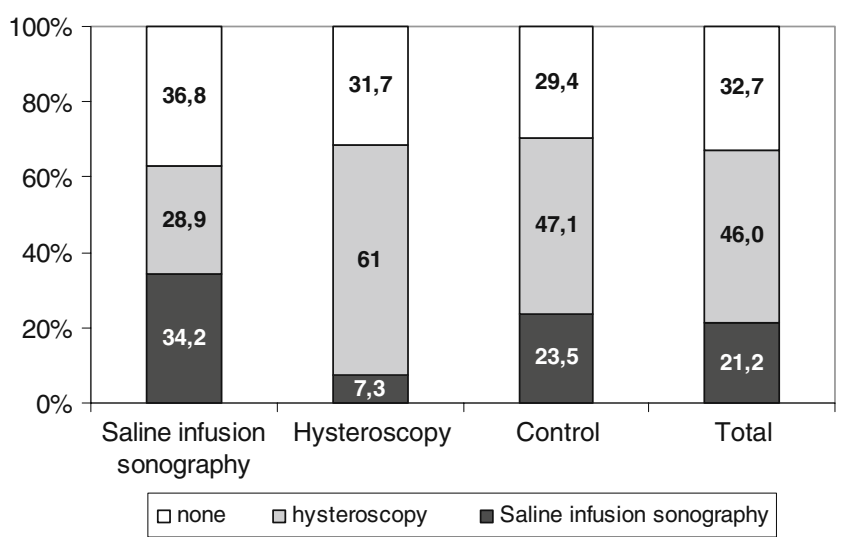

Fig. 2 Preferences in percentages of diagnostic investigation after carefully weighing advantages and disadvantages, stratified by study group. SIS saline infusion sonography

certain advantages of the investigation, sixty-eight (45.1\%) women would accept more pain if the diagnostic investigation and therapy would be offered in one visit. This accounts for $22.7 \%$ of the women preferring SIS and $59.6 \%$ of the women preferring office hysteroscopy $(p=0.02)$. The interviewed women who preferred hysteroscopy would accept hysteroscopy as diagnostic of first choice with a smaller chance of successful therapy than women specifically preferring SIS (Fig. 3).

If during the investigation of the uterine cavity an abnormality would be found, 55 (48.7\%) women would opt for an outpatient treatment, and $38(33.6 \%)$ women would opt for treatment under general anaesthesia. Twenty (15.9\%) women had no preference.

\section{Conclusion}

In this preference study, we found that, given a choice, the majority of women would prefer to undergo office hysteroscopy for further investigation of the uterine cavity. This conclusion may be even stronger with the knowledge that the women participating in this study experienced more pain during office hysteroscopy compared to SIS [9]. In other words, pain scores had no effect on their preferences

Table 2 Mean pain scores (VAS in centimeters) stratified by preference

\begin{tabular}{lllll}
\hline Allocated to & \multicolumn{2}{l}{ Mean pain scores by preference $(95 \%-\mathrm{CI})$} & $p$ \\
\cline { 2 - 4 } & SIS & $\begin{array}{l}\text { Office } \\
\text { hysteroscopy }\end{array}$ & None & \\
\hline SIS & $2.2(0.6-3.8)$ & $2.6(1.2-4.0)$ & $3.5(1.9-5.1)$ & 0.431 \\
$\begin{array}{l}\text { Office } \\
\text { hysteroscopy }\end{array}$ & $6.0(4.0-8.0)$ & $3.1(2.3-3.9)$ & $3.9(3.1-4.7)$ & 0.057 \\
Total & $2.9(1.3-4.5)$ & $3.0(2.2-3.8)$ & $3.7(2.9-4.5)$ & 0.467 \\
\hline
\end{tabular}

Table 3 Reason behind patient preferences for diagnostic investigation of the uterine cavity and subsequent therapy on a Likert scale

Likert score median (25th-75th percentile)

Regarding diagnostic investigation

Least discomfort as possible

$4(3-5)$

Diagnosis and treatment in one

$4(4-5)$

session

Time to consider treatment

$4(3-4)$

options

Small risk failure investigation

$4(4-5)$

Regarding therapy

Short waiting time therapy $\quad 5(4-5)$

Small surgery risk $\quad 5(4-5)$

Anaesthesia during therapy $3(3-4)$

No anaesthesia during therapy $3(3-4)$

High chance therapy successful $5(5-5)$

1 very unimportant, 2 unimportant, 3 neutral, 4 important, 5 very important

afterwards. In women preferring SIS for uterine cavity assessment, the most important factor for their preference seems to be pain, while in women preferring hysteroscopy, other factors (e.g. immediate therapy) seemed to be of more importance in their preference.

A limitation of this study remains that we had to design a questionnaire specifically for this purpose because there were no validated questionnaires available. In order to minimise bias due to experience, we included women randomly allocated to SIS and office hysteroscopy who presumably had no strong preference beforehand and women with no history of uterine cavity investigation, which makes our design unique.

Another possible source of bias results from what is called cognitive justification - a phenomenon that makes people

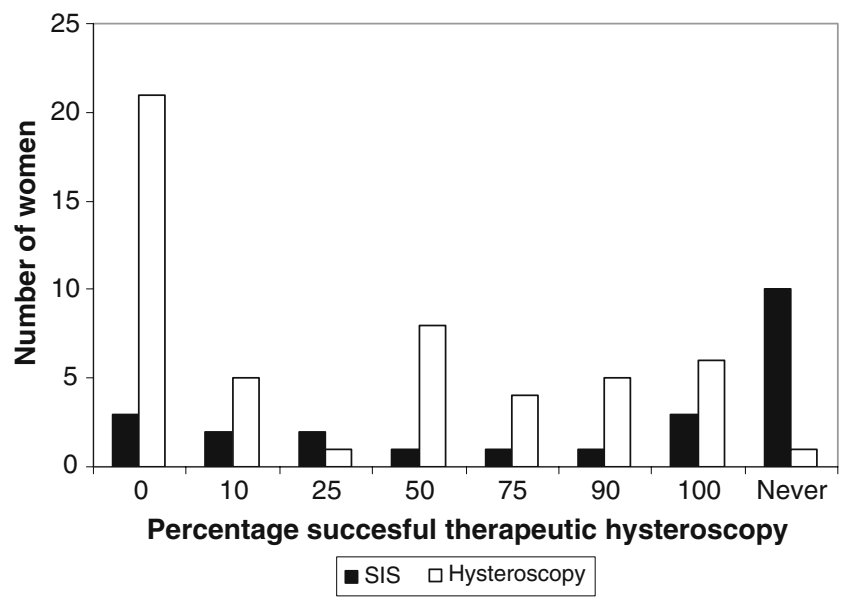

Fig. 3 Acceptance of hysteroscopy by the percentage of required successful hysteroscopic therapy among women preferring SIS and hysteroscopy. SIS saline infusion sonography 
change their perceptions to make their situation seem better. Women were included in this study after undergoing SIS or office hysteroscopy, which might explain why the preference for the treatment they underwent was strongest. However, this accounts for both SIS and office hysteroscopy. Moreover, it is interesting that women of the control group preferred office hysteroscopy over SIS.

The most important reason for this preference was completeness of diagnosis and therapy in one visit. Additionally, our study showed that, apart from the diagnostic investigation, most women would prefer to undergo therapy in an outpatient setting rather than a day case setting. This is in concordance with previously published literature $[7,8,10]$. As proposed by Marsh et al. [8], we included in our questionnaire information about the risks and side effects of general anaesthesia in order to investigate whether the need for regional or general anaesthesia with day case hysteroscopy would influence their preference for an outpatient or day case approach. Surprisingly, this was not regarded of any importance in their preference.

Furthermore, most women prefer being informed about all advantages and disadvantages of diagnostic and therapeutic possibilities in general and prefer to participate in this decision. Considering this and the fact that a considerable part of the respondents preferred SIS and treatment under general anaesthesia, women should be informed of their options completely and not automatically be offered office hysteroscopy as the 'preferred' choice of investigation. Especially since the prevalence of intrauterine abnormalities after selection by transvaginal ultrasound examination is only $50 \%$ [11], and we do not know yet what the success rates are for treatment by office hysteroscopy.

In conclusion, we found that, given the choice, the majority of women would prefer office hysteroscopy over SIS. Additionally, if there was an indication for intrauterine surgery, an outpatient setting is preferred to a day case setting. Nevertheless, one third of the surveyed women would rather be treated under general anaesthesia. So, although our results support the establishment of outpatient one-stop clinics, the inpatient alternative should be offered as well.

Conflict of interest The authors report no conflict of interest. The authors alone are responsible for the content and writing of the paper.

Open Access This article is distributed under the terms of the Creative Commons Attribution Noncommercial License which permits any noncommercial use, distribution, and reproduction in any medium, provided the original author(s) and source are credited.

\section{Appendix A}

Table 4 The descriptions of saline infusion sonography and office hysteroscopy

Saline infusion sonography

In general

This procedure is used to determine the presence or absence of abnormalities in the uterine cavity.

Description of procedure

The patient is seated in an examination chair with the legs resting on two knee supports. The doctor or investigator places a speculum in the vagina. A thin catheter (cross-sectional plane, $2 \mathrm{~mm}$ ) is inserted through the neck of the womb into the womb, through which sterile normal saline solution is injected. When filled, a vaginal ultrasound is performed.

Duration of procedure

15 min. Directly after the procedure, the patient may go home.

Anaesthesia

None

Therapy

If an abnormality is found ( $50 \%$ of cases), a new appointment to treat it
Office hysteroscopy

In general

This procedure is used to determine the presence or absence of abnormalities in the uterine cavity. Occasionally during this procedure, treatment will immediately follow a diagnosis.

Description of procedure

The patient is seated on an examination chair with the legs resting on two knee supports. The doctor inserts a thin telescope (cross-sectional plane, $4 \mathrm{~mm}$ ) through the vagina and neck of the womb, into the womb (without using a speculum). Through the telescope, sterile saline solution is injected into the womb. Once the tip of the hysteroscope is in the womb, the inner wall is seen on a TV screen and can be evaluated.

Duration of procedure

$15 \mathrm{~min}$. If subsequent therapy is required, an additional 15-30 min. Directly after the procedure, the patient may go home.

Anaesthesia

None

Therapy 
Table 4 (continued)

Saline infusion sonography

will be made. Depending on the type of abnormality, treatment will take place in the outpatient clinic by hysteroscopy (as described on the right). If this is not possible, treatment under general anaesthesia in the operating room may be required.

\section{Risk}

Complications of SIS, $0.2 \%$ (1 out of 500 ; e.g. infection)

Failure of procedure In $16 \%$ (16 out of 100$)$ of the cases, the procedure will fail or will not provide enough information on the suspected pathology. In such cases, a new appointment will be made to perform a hysteroscopy in the outpatient clinic.
Office hysteroscopy

doctor will be able to remove it with a special instrument introduced through the telescope, e.g. removal of polyps or by taking biopsies for further analysis. If this is not possible, treatment under general anaesthesia in the operating room may be required.

Risk

Complications of hysteroscopy, $0.4 \%$ ( 1 out of 250 ; e.g. infection, bleeding)

Failure of procedure

In $12 \%$ (12 out of 100) of the cases, the procedure will fail or will not provide enough information on the suspected pathology. In such cases, the hysteroscopy will be repeated under general anaesthesia in the operating room.

\section{References}

1. Clark TJ, Voit D, Gupta JK, Hyde C, Song F, Khan KS (2002) Accuracy of hysteroscopy in the diagnosis of endometrial cancer and hyperplasia: a systematic quantitative review. JAMA 288:1610-1621

2. van Dongen H, de Kroon CD, Jacobi CE, Trimbos JB, Jansen FW (2007) Diagnostic hysteroscopy in abnormal uterine bleeding: a systematic review and meta-analysis. BJOG 114:664-675

3. de Kroon CD, de Bock GH, Dieben SW, Jansen FW (2003) Saline contrast hysterosonography in abnormal uterine bleeding: a systematic review and meta-analysis. BJOG 110:938-947

4. Rogerson L, Bates J, Weston M, Duffy S (2002) A comparison of outpatient hysteroscopy with saline infusion hysterosonography. BJOG 109:800-804

5. Widrich T, Bradley LD, Mitchinson AR, Collins RL (1996) Comparison of saline infusion sonography with office hysteroscopy for the evaluation of the endometrium. Am J Obstet Gynecol 174:1327-1334
6. Bettocchi S, Selvaggi L (1997) A vaginoscopic approach to reduce the pain of office hysteroscopy. J Am Assoc Gynecol Laparosc 4:255-258

7. Kremer C, Duffy S, Moroney M (2000) Patient satisfaction with outpatient hysteroscopy versus day case hysteroscopy: randomised controlled trial. BMJ 320:279-282

8. Marsh F, Taylor L, Kremer C, Black J, Duffy S (2002) Delivering an effective outpatient service in gynaecology: an assessment of patient preference. Gynaecol Endosc 11:337-343

9. van Dongen $\mathrm{H}$, de Kroon $\mathrm{CD}$, van den Tillaart S, Louwe $\mathrm{L}$, Trimbos-Kemper T, Jansen FW (2008) A randomized comparison of office vaginoscopic hysteroscopy with saline infusion sonography: a patient compliance study. BJOG 115:1232-1237

10. Ferry J, Rankin L (1994) Low cost, patient acceptable, local analgesia approach to gynaecological outpatient surgery. A review of 817 consecutive procedures. Aust N Z J Obstet Gynaecol 34:453-456

11. Towbin NA, Gviazda IM, March CM (1996) Office hysteroscopy versus transvaginal ultrasonography in the evaluation of patients with excessive uterine bleeding. Am J Obstet Gynecol 174:1678-1682 